\title{
Numerical shock propagation using geometrical shock dynamics
}

\author{
By W. D. HENSHA W†, N. F. SMYTH \\ AND D. W. SCHWENDEMAN \\ Applied Mathematics Department, California Institute of Technology, \\ Pasadena CA 91125, USA
}

(Received 2 August 1985 and in revised form 21 January 1986)

A simple numerical scheme for the calculation of the motion of shock waves in gases based on Whitham's theory of geometrical shock dynamics is developed. This scheme is used to study the propagation of shock waves along walls and in channels and the self-focusing of initially curved shockfronts. The numerical results are compared with exact and numerical solutions of the geometrical-shock-dynamics equations and with recent experimental investigations.

\section{Introduction}

The numerical solution of hyperbolic differential equations and the associated problem of numerical shock fitting has received much attention in the recent literature. The main numerical difficulty in their solution lies in the method of fitting the shock. The determination of the motion of the shock requires the calculation of the flow field in some region around the shock. In many problems, one is interested in the motion of the shock alone and not the details of the flow field. Also, most of the numerical work has dealt with smooth shocks, leaving open the more difficult problem of situations in which the shock becomes non-smooth, as in Mach reflection.

In the present work, we shall be interested in the numerical propagation of shock waves in gases. Rather than solve the gasdynamic equations directly, the motion of the shock will be determined using geometrical shock dynamics, as developed by Whitham $(1957,1959)$. By using this approximate theory, the motion of the shock can be determined without explicitly calculating the flow field behind. Furthermore, the equations of geometrical shock dynamics are nonlinear and hyberbolic; thus, discontinuities in the shockfront appear naturally as shock-shocks. We briefly discuss the main elements of the theory of geometrical shock dynamics in $\$ 2$.

Geometrical shock dynamics propagates a shock along rays normal to the shockfront with the local speed of propagation depending on the local Mach number. Numerically, this procedure is performed by representing the shockfront by a discrete set of points and propagating each point along approximate normals with a speed determined by the discrete Mach number-area relation. In expansive regions of the shockfront, points are automatically inserted to maintain a good shockfront resolution. Shock-shocks are fitted in compressive regions of the shockfront by deleting points. A smoothing scheme is added to dampen high-frequency numerical fluctuations in the shockfront. The overall procedure is performed rapidly

$\dagger$ Present address: International Business Machines Corporation, Thomas J. Watson Research Center, P.O. Box 218, Yorktown Heights, NY 10598, USA.

$\ddagger$ Present address: Department of Mathematics, University of New South Wales, Kensington, N.S.W. 2033, Australia. 
with good accuracy for a wide variety of problems. The details of this numerical scheme are presented in $§ 3$.

The method of geometrical shock dynamics is not limited to shock waves and can be used for the propagation of any wavefront for which there is a known relation between the local speed of propagation and the local amplitude. For example, Miles (1977) applied geometrical shock dynamics to the propagation of a soliton around corners. The numerical method presented here can also be used for these problems.

In $\S 4$, we compare results obtained using our numerical scheme with exact and numerical solutions and experimental data. We consider the cases of shock-wave diffraction, shock waves in channels and focusing shock waves.

Exact solutions using geometrical shock dynamics were found by Whitham for some shock-wave diffraction problems. Results calculated numerically are compared with these exact solutions. Bryson \& Gross (1961) obtained numerical and experimental results for shock-wave diffraction by cones, cylinders and spheres. Their numerical results used the method of characteristics, in contrast to the scheme we present in §3. Shockfronts calculated using our numerical scheme are compared with these results as well.

Edwards, Fearnley \& Nettleton (1983) experimentally studied the propagation of initially plane shock waves in channels with a $90^{\circ}$ circular bend. Results were obtained for the Mach number on the convex and concave walls and a description of the shock geometry was discussed. We compare our numerical calculations with their results.

Sturtevant \& Kulkarny (1976) experimentally determined the behaviour of focusing weak shock waves by reflecting plane shock waves from a concave wall. Depending on the initial Mach number and curvature of the wall, the shock either focused to form a crossed and folded front similar to that which occurs for geometrical acoustics or the shock remained uncrossed. We find that the approximate theory of geometrical shock dynamies is able to predict this observation with reasonable accuracy.

\section{Geometrical shock dynamics}

Whitham $(1957,1959)$ developed an approximate theory for shock propagation analagous to geometrical acoustics. In this theory, called geometrical shock dynamics, the shock propagates on rays normal to the shockfront, with the speed of propagation depending on the local amplitude of the shock. This theory is summarized in Whitham (1974, chapter 8).

Whitham shows that the local Mach number and ray-tube area are related by

$$
A=A_{0} \frac{f(M)}{f\left(M_{0}\right)},
$$

where $M_{0}$ and $A_{0}$ are the initial Mach number and ray-tube area respectively. The function $f(M)$ is given by

$$
f(M)=\exp \left(-\int \frac{M \lambda(M)}{M^{2}-1} \mathrm{~d} M\right)
$$

where

$$
\begin{aligned}
\lambda(M) & =\left(1+\frac{2}{\gamma+1} \frac{1-\mu^{2}}{\mu}\right)\left(1+2 \mu+\frac{1}{M^{2}}\right), \\
\mu^{2} & =\frac{(\gamma-1) M^{2}+2}{2 \gamma M^{2}-(\gamma-1)},
\end{aligned}
$$




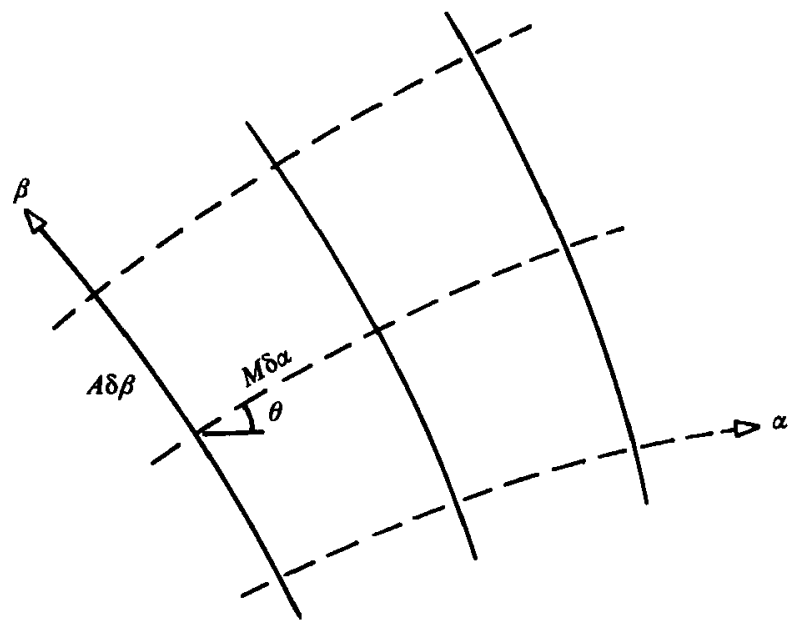

Figure 1. Coordinate system $(\alpha, \beta)$. Shock positions given by $\alpha=$ const (solid lines) and rays given by $\beta=$ const (dashed lines).

$\gamma$ being the ratio of specific heats $(=1.4$ for air). For clarity, we shall refer to (2.1) as the $A-M$ relation. The integral in expression (2.2) may be evaluated explicitly. However, a numerical evaluation is more convenient. Approximations to (2.2) may also be obtained in the limits of weak and strong shocks. For weak shocks

$$
f(M)=(M-1)^{-2} \text { as } M \rightarrow 1,
$$

and for strong shocks

$$
f(M)=M^{-n} \quad \text { as } M \rightarrow \infty,
$$

where $n=1+(2 / \gamma)+(2 \gamma / \gamma-1)^{\frac{1}{2}}=5.0743$ for $\gamma=1.4$.

In the present work, we shall deal primarily with two-dimensional motion, even though the theory of shock dynamics applies in three dimensions as well. The two-dimensional formulation may be extended to axisymmetric motion without much change and we treat some of these cases also.

In two dimensions, it is convenient to introduce an orthogonal curvilinear coordinate system $(\alpha, \beta)$ where successive shock positions are described by curves $\alpha=$ constant and rays by $\beta=$ constant (figure 1 ). The shockfront is described by its local Mach number $M(\alpha, \beta)$ and its ray inclination angle $\theta(\alpha, \beta)$ referenced to a fixed direction, the $x$-axis say. It follows from a geometrical argument that the system of equations describing the propagation of the shockfront is

$$
\left.\begin{array}{c}
\frac{\partial \theta}{\partial \beta}-\frac{A^{\prime}(M)}{M} \frac{\partial M}{\partial \alpha}=0 \\
\frac{\partial \theta}{\partial \alpha}+\frac{1}{A(M)} \frac{\partial M}{\partial \beta}=0
\end{array}\right\}
$$

where $A=A(M)$ is the $A-M$ relation. The characteristie form of $(2.5)$ is

$$
\left(\frac{\partial}{\partial \alpha} \pm c \frac{\partial}{\partial \beta}\right)(\theta \pm \omega(M))=0
$$

where $\quad c^{2}(M)=-\frac{M}{A A^{\prime}}>0, \quad \omega(M)=\int_{1}^{M} \frac{\mathrm{d} M}{A c}=\int_{1}^{M}\left(\frac{\lambda(M)}{M^{2}-1}\right)^{\frac{1}{2}} \mathrm{~d} M$. 
(a)

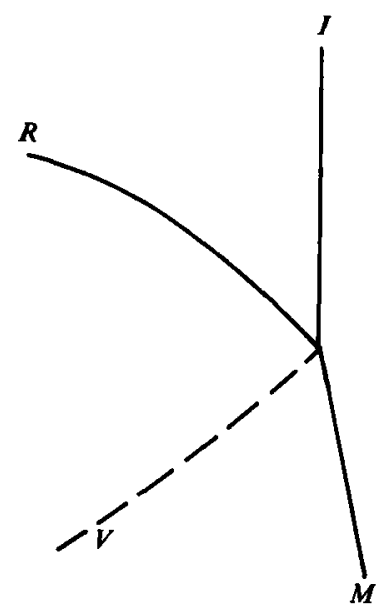

(b)

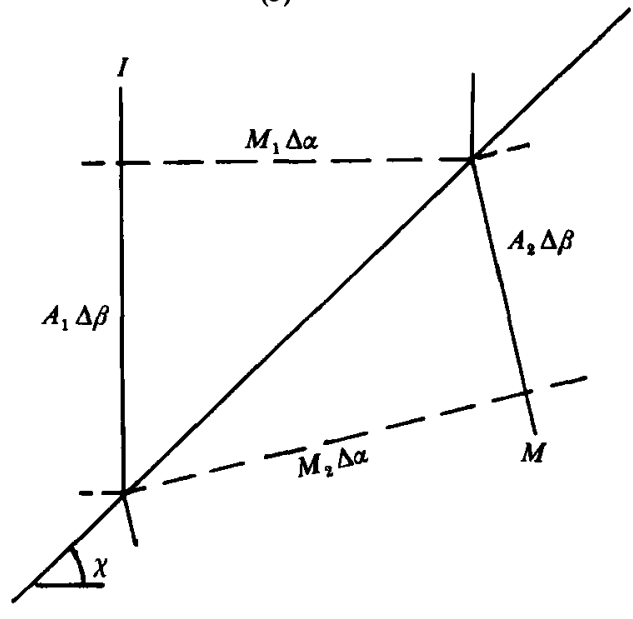

Figure 2. (a) Mach reflection: incident shock $I$, Mach stem $M$, reflected shock $R$ and vortex sheet $V$. (b) Shock-shock geometry. Constant Mach number and ray inclination angle at state 1 separated from state 2 by shock-shock line inclined with angle $\chi$.

Therefore,

$$
\theta \pm \omega(M)=\text { constant on characteristics } C_{ \pm}: \frac{\mathrm{d} \beta}{\mathrm{d} \alpha}= \pm c .
$$

The system (2.5) is hyperbolic and represents a wave motion for disturbances propagating on the shock. Since this system of equations is hyperbolic and nonlinear, the shockfronts can develop discontinuities in slope and Mach number, these being called shock-shocks. Shock-shocks correspond physically to the formation of Mach stems, the shock-shock discontinuity being the position of the triple-point. In Mach reflection there is also a reflected shock $R$ and a vortex sheet $V$, as shown in figure $2(a)$, but they do not appear explicitly in geometrical shock dynamics. Only the leading fronts appear, as in figure $2(b)$. The change in direction and Mach number at the shock-shock imply their existence.

Where a shock-shock develops, it is possible to treat it as a discontinuity in $M$ and $\theta$. Whitham derives the appropriate jump conditions relating $M_{1}$ and $\theta_{1}$ ahead of the shock-shock with $M_{2}$ and $\theta_{2}$ behind it in terms of $\chi$, the angle of the shock-shock line with the $x$-axis (figure $2 b$ ). These jump relations are

$$
\begin{gathered}
\tan \left(\theta_{2}-\theta_{1}\right)=\frac{\left(M_{2}^{2}-M_{1}^{2}\right)^{\frac{1}{2}}\left(A^{2}\left(M_{1}\right)-A^{2}\left(M_{2}\right)\right)^{\frac{1}{2}}}{A\left(M_{2}\right) M_{2}+A\left(M_{1}\right) M_{1}} . \\
\tan \left(\chi-\theta_{i}\right)=\frac{A\left(M_{i}\right)}{M_{i}}\left(\frac{M_{2}^{2}-M_{1}^{2}}{A^{2}\left(M_{1}\right)-A^{2}\left(M_{2}\right)}\right)^{\frac{1}{2}}, \quad i=1 \text { or } 2 .
\end{gathered}
$$

Assuming $M_{1}$ and $\theta_{1}$ are known, two of the three remaining variables are given by (2.8) and (2.9) if one of the remaining is known. In the diffraction problems considered later, $\theta_{2}$ is known from the geometry and this then gives $M_{2}$ and $\chi$.

The characteristic solution (2.7) along with jump conditions (2.8) and (2.9) may be used to construct solutions in some simple cases. Solutions found in this manner are completely analogous to solutions found in one-dimensional gasdynamics or shallow-water waves for example. Later, the accuracy of the approximate solution obtained numerically will be checked with some of these exact solutions. 


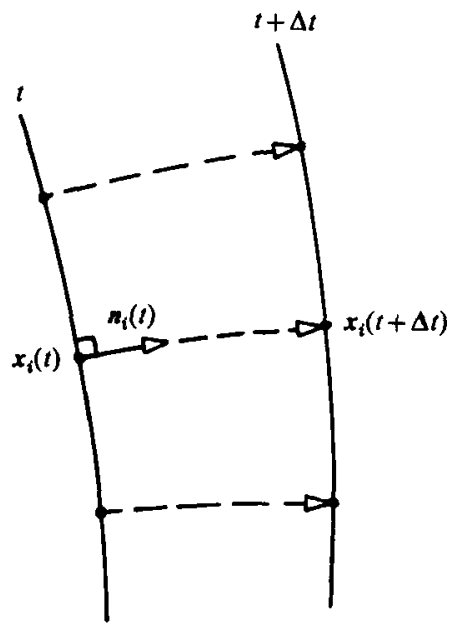

Figure 3. Time-marching scheme. Shockfront positions at $t$ and $t+\Delta t$ (solid lines) and approximate rays (dashed lines).

\section{Numerical scheme}

In this section, we present the numerical scheme developed to calculate successive shockfronts using the theory of geometrical shock dynamics. The scheme is advantageous in its simplicity, which will become clear shortly, and its application to a wide variety of problems. Later, we shall apply the numerical procedure to calculate shocks for many cases.

The numerical procedure is basically a leap-frog time-marching scheme. A sketch of the time-marching scheme is provided in figure 3 . The shockfront is given by a discrete set of points. Each point is advanced along its normal with a speed specified by the discrete version of the $A-M$ relation. In expansive regions of the shockfront, we insert points in order to maintain an approximately uniform point distribution. In compressive regions, we fit shock-shocks into the front by deleting points. A smoothing procedure is employed to dampen high-frequency numerical errors in the shockfront position.

The numerical scheme is based on an approximate integration along rays. Un a ray

$$
\frac{\partial x}{\partial \alpha}=M \cos \theta, \quad \frac{\partial y}{\partial \alpha}=M \sin \theta
$$

We write (3.1) in vector form and eliminate $\alpha$ in favour of time $t$ using $\alpha=a_{0} t$ where $a_{0}$ is some undisturbed sound speed. Equations (3.1) become

$$
\frac{\partial}{\partial t} \boldsymbol{x}(\beta, t)=a_{0} M(\beta, t) n(\beta, t)
$$

where $x=(x, y)$ is the shockfront position and $n=(\cos \theta, \sin \theta)$ is the normal to the shockfront.

We approximate the shockfront position by a discrete set of points $x_{j}(t), j=1$, $\ldots, N$. Let $M_{i}(t)$ and $n_{i}(t)$ approximate the Mach number and shockfront normal at $x_{i}(t)$ respectively. Then, (3.2) becomes

$$
\frac{\mathrm{d}}{\mathrm{d} t} \boldsymbol{x}_{i}(t)=M_{i}(t) n_{i}(t), \quad i=1, \ldots, N,
$$


where we set $a_{0}=1$ in all our calculations. System (3.3) is a nonlinear system of ODEs and may be numerically integrated in a number of ways. We choose to use a two-step leap-frog scheme

$$
\boldsymbol{x}_{i}(t+\Delta t)=\boldsymbol{x}_{i}(t-\Delta t)+2 \Delta t M_{i}(t) \boldsymbol{n}_{i}(t), \quad i=1, \ldots, N,
$$

where $t=n \Delta t, n=0, \ldots, T / \Delta t$. Scheme (3.4) is explicit and second-order accurate in time. Furthermore, leap-frog adds no numerical dissipation which is a desirable property since system (3.2) is hyperbolic.

The Mach number $M_{i}(t)$ in (3.4) is found by solving the approximate $A-M$ relation

$$
\frac{A_{i}(t)}{A_{i}(0)}=\frac{f\left(M_{i}(t)\right)}{f\left(M_{i}(0)\right)} \quad \text { for } i=1, \ldots, N .
$$

Inverting the function $f(M)$ gives

$$
M_{i}(t)=f^{-1}\left(f\left(M_{i}(0)\right) \frac{A_{i}(t)}{A_{i}(0)}\right) \quad \text { for } i=1, \ldots, N
$$

The inverse function $f^{-1}$ may be found explicitly in the limit of weak shocks (2.3) or strong shocks (2.4); however, we solve (3.6) numerically in general. The approximate area $A_{i}(t)$ in (3.6) is given by a centred scheme about the point $x_{i}(t)$ in the interior and $a$ one-sided scheme at the endpoints

$$
A_{i}(t)=\frac{1}{2} \begin{cases}s_{i+1}(t)-s_{i}(t), & \text { if } i=1 \\ s_{i+1}(t)-s_{i-1}(t), & \text { if } i=2, \ldots, N-1 \\ s_{i}(t)-s_{i-1}(t), & \text { if } i=N\end{cases}
$$

where $s_{i}(t)$ is the discrete arclength given by

$$
s_{i}(t)= \begin{cases}0, & \text { if } i=1 ; \\ s_{i-1}(t)+\left|x_{i}(t)-x_{i-1}(t)\right|, & \text { if } i=2, \ldots, N .\end{cases}
$$

For the case of axisymmetric flow, we need only change the definition of $A_{i}(t)$ in the numerical scheme. The approximate area in this case is

$$
A_{i}(t)=\frac{1}{2} \pi \begin{cases}\left(y_{i+1}+y_{i}\right)\left(s_{i+1}-s_{i}\right), & \text { if } i=1 \\ 2 y_{i}\left(s_{i+1}-s_{i-1}\right), & \text { if } i=2, \ldots, N-1 \\ \left(y_{i}+y_{i-1}\right)\left(s_{i}-s_{i-1}\right), & \text { if } i=N .\end{cases}
$$

The normal to the shockfront $n(t)$, which is required in (3.4), is determined by differentiating two cubic splines fitted to the data $\left(s_{j}(t), x_{j}(t)\right)$ and $\left(s_{j}(t), y_{j}(t)\right)$, $j=1, \ldots, N$. Let $\tilde{x}(s)$ and $\tilde{y}(s)$ denote these two cubic spline interpolants respectively. The smooth curve $(\tilde{x}(s), \tilde{y}(s))$ is an approximation to the shockfront at time $t$. Therefore, $n_{i}(t)$ is given by

$$
\boldsymbol{n}_{i}(t)=\frac{\left(\tilde{y}^{\prime}\left(s_{i}\right),-\tilde{x}^{\prime}\left(s_{i}\right)\right)}{\left[\left(\tilde{x}^{\prime}\left(s_{i}\right)\right)^{2}+\left(\tilde{y}^{\prime}\left(s_{i}\right)\right)^{2}\right]^{\frac{1}{2}}}, \quad i=\mathbf{1}, \ldots, N,
$$

where the primes denote differentiation with respect to $s$. 
The shockfront position $\boldsymbol{x}_{i}(0)$ and the Mach number $M_{i}(0)$ are determined by the initial data given at $t=0$. The parameters $N$ and $\Delta t$ are chosen by the general rules:

Rule 1

$$
\Delta s_{\mathrm{avg}}=\frac{1}{N} \sum_{i=2}^{N} \Delta s_{i}(0)=\frac{s_{N}(0)}{N}=K_{1} \ll 1,
$$

Rule 2

$$
\frac{\Delta t}{\Delta s_{\min }}=\frac{\Delta t}{\min _{\substack{2 \leqslant i \leqslant N \\ 0 \leqslant t \leqslant T}} \Delta s_{i}(t)}=\frac{\Delta t}{d \Delta s_{\mathrm{avg}}}<K_{2}=O(1),
$$

where $\Delta s_{i}(t)=s_{i}(t)-s_{i-1}(t)$ and $d$ is a minimum tolerance on $\Delta s_{i}(t)$, which we discuss in detail later. Usually we take constants $K_{1}$ and $K_{2}$ equal to 0.01 and 0.2 respectively. Rule 1 (3.11) provides an adequate resolution of the shockfront. Rule 2 (3.12) is the Courant-Friedrichs-Lewy condition and gives stability for all cases run. The shockfront at $t=\Delta t$ is determined using a second-order one-step explicit scheme which provides the necessary initial information to begin the two-step leap-frog scheme.

The numerical scheme as described previously may be used in the absence of wall boundaries. These pure initial-value problems occur in the case of self-focusing shock waves, for example.

In the presence of wall boundaries, the appropriate boundary conditions must be applied. Walls coincide with rays in the theory of geometrical shock dynamics; therefore, at wall boundaries the shock must be normal to the walls. Figure 4 illustrates how this boundary condition is implemented numerically. Points interior to boundaries are calculated using the leap-frog scheme. The point at the boundary is then determined such that the line segment between the endpoint and its neighbouring internal point is normal to the wall. Examples of these initial-boundaryvalue problems are shock-wave diffraction and shock waves in channels.

The numerical scheme provides for the insertion and deletion of points as they tend to spread out in expansive regions or cluster in compressive regions of the shockfront. The point spacing $\Delta s_{i}(t)$ is checked periodically and we require

$$
d \leqslant \frac{\Delta s_{i}(t)}{\Delta s_{\mathrm{avg}}} \equiv \sigma_{i}(t) \leqslant D \quad \text { for all } i=2, \ldots, N,
$$

where $d=\frac{1}{2}$ and $D=\frac{3}{2}$ usually. If $\sigma_{i}(t)<d$, point $x_{i}(t)$ is removed and if $\sigma_{i}(t)>D$, point $\boldsymbol{x}_{i-\frac{1}{2}}(t)$ is added using the cubic-spline interpolant evaluated at $\frac{1}{2}\left(s_{i}(t)+s_{i-1}(t)\right)$. We preserve the area ratio $A_{i}(t) / A_{i}(0)$ in (3.6) by removing or adding the points at $t=0$ as well. In either case if (3.13) fails the leap-frog scheme is restarted as previously described for $t=0$.

We find it desirable to insert points in order to maintain the initially prescribed shockfront resolution (3.11). Deleting points ensures numerical stability at each time step (3.12). Furthermore, rays tend to cross as shock-shocks form in compressive regions of the shockfront. By deleting points, shock-shocks are effectively fitted into the shockfront.

A simple two-step smoothing procedure is added to the numerical scheme in order to dampen the high-frequency errors in $x_{i}(t)$. After every $n_{\mathrm{s}}$ time steps (usually 10 to 50) we let

$$
\frac{1}{2}\left(x_{i+1}(t)+x_{i-1}(t)\right) \rightarrow x_{i}(t),
$$

where we scan $i$-even then $i$-odd for $1<i<N$. The numerical scheme is then 


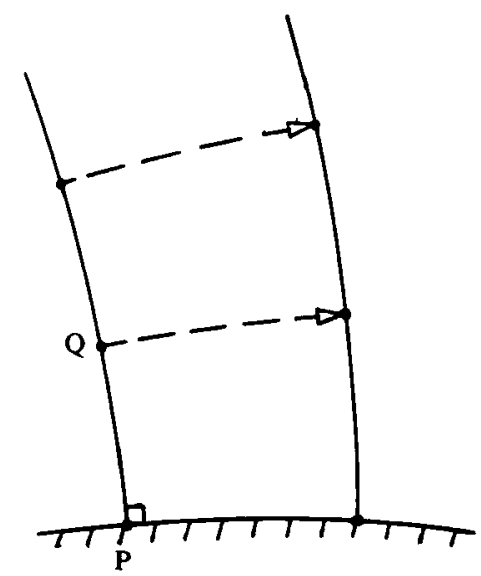

Figure 4. Numerical boundary conditions at a wall. Line segment $P Q$ is normal to the wall at point $P$.

restarted using the smoothed shockfront as initial conditions. The smoothing scheme (3.14) is one Jacobi iteration applied to the discrete system of equations

$$
D^{2} x_{i}(t) \equiv x_{i-1}(t)-2 x_{i}(t)+x_{i+1}(t)=0, \quad 1<i<N
$$

This iteration is effective in damping high-frequency fluctuations in $x_{i}(t)$. The overall shockfront given by the lower frequencies is essentially unchanged by this procedure.

\section{Results and discussion}

Successive shockfront positions are calculated numerically for the cases of shockwave diffraction, shock waves in channels and focusing shock waves. We establish the accuracy of our numerical scheme by comparing solutions obtained numerically with exact solutions found by Whitham and with numerical solutions found by Bryson \& Gross (1961) using the method of characteristies for shock-wave diffraction. We then compare the numerical calculations with experimental data for all three cases. Many important features of the flows presented in the experiments, namely shockfront positions, triple-point positions (i.e. shock-shocks) and wall Mach numbers, are well represented in the numerical calculations. This demonstrates the usefulness of the geometrical-shock-dynamics approximation.

\subsection{Shock-wave diffraction}

A simple solution may be constructed using characteristics (2.7) for shock-wave diffraction by a convex wall (Whitham 1974). On a $C_{-}$characteristic

$$
\theta-\omega(M)=\text { const. }
$$

Since all $C_{-}$characteristics originate from the undisturbed region, $\theta=0$ and $M=M_{0}$, we have

$$
\theta-\omega(M)=-\omega\left(M_{0}\right)=\text { const everywhere. }
$$

In particular, the wall Mach number $M_{\mathrm{w}}$, perhaps the most important quantity for this problem, may be found given the wall inclination angle $\theta_{w}$. Using (4.2) at the wall gives

$$
\omega\left(M_{\mathrm{w}}\right)=\theta_{\mathrm{w}}+\omega\left(\boldsymbol{M}_{\mathbf{0}}\right)
$$



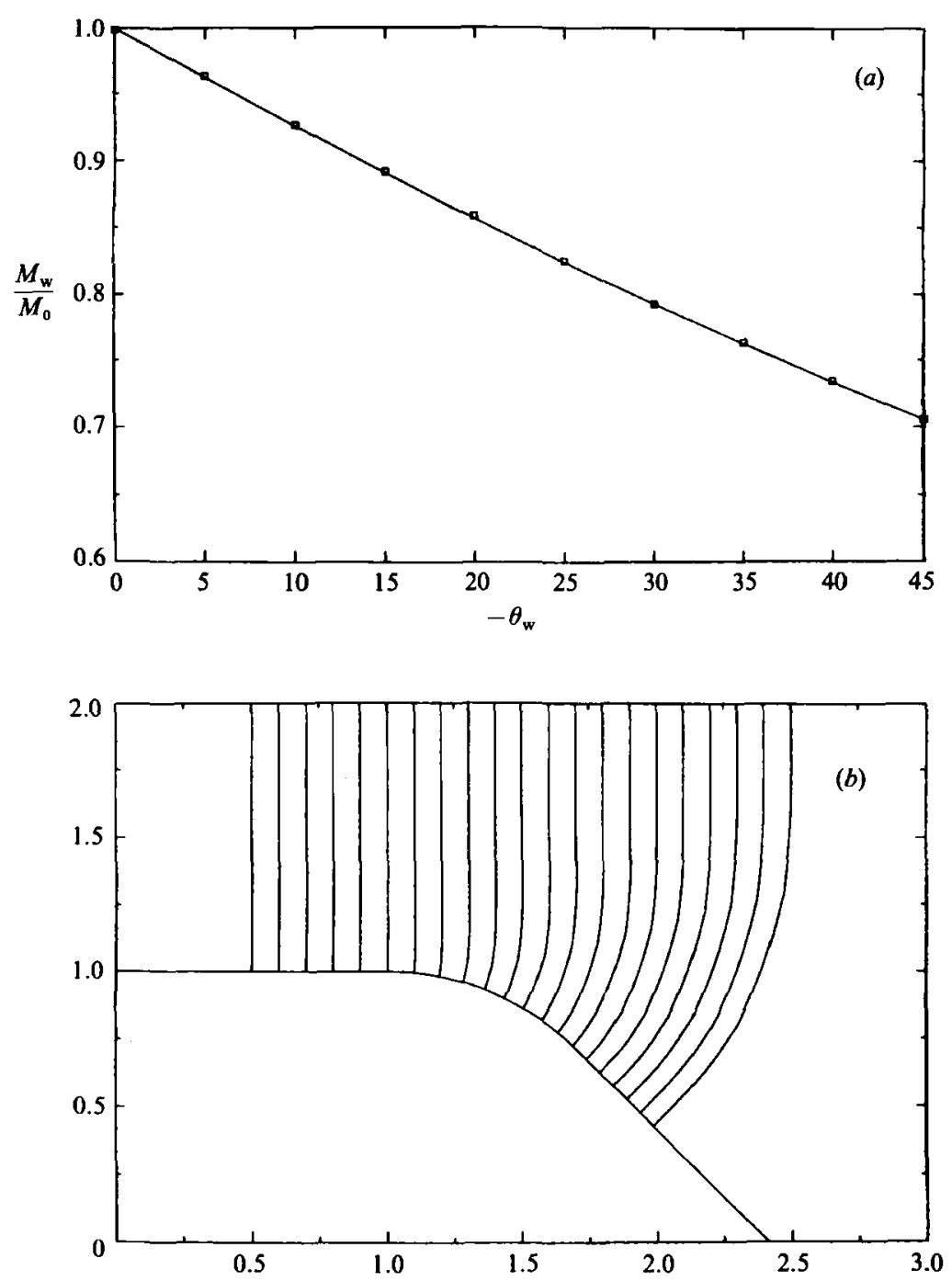

Figure 5. Diffraction by a convex wall: $(a) M_{w} / M_{0} v s .-\theta_{w} ;(b)$ expanding shockfronts around a $45^{\circ}$ circular convex wall.

For the purpose of testing our numerical scheme we use the strong-shock approximation (2.4) which gives $M_{\mathrm{w}}$ explicitly:

$$
M_{\mathrm{w}}=M_{0} \mathrm{e}^{k \theta_{\mathrm{w}}}
$$

where $k=n^{-\frac{1}{2}}=0.44393$ for $\gamma=1.4$. Whitham goes on to complete the solution for this problem; however, we stop here since we are primarily interested in comparing $M_{\mathrm{w}}$.

In figure $5(a)$ we plot the value of $M_{\mathrm{w}} / M_{0}$ vs. $-\theta_{\mathrm{w}}$ using (4.4). The expanding shockfronts around a $45^{\circ}$ convex wall are calculated numerically and shown in figure $5(b)$. From our calculations, we determine $M_{w} / M_{0}$ at evenly spaced intervals of time and plot these values versus $-\theta_{\mathrm{w}}$ as well. We see that the agreement between the exact curve and the numerical values is good.

For shock-wave diffraction by a wedge it is also possible to obtain an exact 
solution. Here we use the jump conditions (2.8) and (2.9) with $M_{1}=M_{0}, \theta_{1}=0$ and $M_{2}=M_{\mathrm{w}}, \theta_{2}=\theta_{\mathrm{w}}$

$$
\begin{gathered}
\tan \theta_{\mathrm{w}}=\frac{\left(M_{\mathrm{w}}^{2}-M_{0}^{2}\right)^{\frac{1}{2}}\left(A^{2}\left(M_{0}\right)-A^{2}\left(M_{\mathrm{w}}\right)\right)^{\frac{1}{2}}}{A\left(M_{\mathrm{w}}\right) M_{\mathrm{w}}+A\left(M_{0}\right) M_{0}}, \\
\tan \left(\chi-\theta_{\mathrm{w}}\right)=\frac{A\left(M_{\mathrm{w}}\right)}{M_{\mathrm{w}}}\left(\frac{M_{\mathrm{w}}^{2}-M_{0}^{2}}{A^{2}\left(M_{0}\right)-A^{2}\left(M_{\mathrm{w}}\right)}\right)^{\frac{1}{2}},
\end{gathered}
$$

where $\chi$ is the angle of the shock-shock line. Again we use the strong-shock approximation which gives

$$
\begin{aligned}
& \tan \theta_{w}=\frac{\left(r^{-2}-1\right)^{\frac{1}{2}}\left(1-r^{2 n}\right)^{\frac{1}{2}}}{r^{n-1}+1} \\
& \tan \left(\chi-\theta_{w}\right)=r^{n}\left(\frac{1-r^{2}}{1-r^{2 n}}\right)^{\frac{1}{2}}
\end{aligned}
$$

where $r=M_{0} / M_{w}$. Equation (4.7) gives $r$ implicitly in terms of $\theta_{w}$. Equation (4.8) then gives $\chi=\chi\left(\theta_{\mathrm{w}}\right)$.

We plot $\chi\left(\theta_{\mathrm{w}}\right)-\theta_{\mathrm{w}}$ vs. $\theta_{\mathrm{w}}$ in figure $6(a)$. Successive shockfronts are calculated for $\theta_{\mathrm{w}}=10^{\circ}, 20^{\circ}, \ldots, 70^{\circ}$ and $\chi$ is determined for each from their graphs. Again we note the good agreement with the exact solution for all values of $\theta_{\mathrm{w}}$. A representative calculation for $\theta_{\mathrm{w}}=30^{\circ}$ is displayed in figure $6(b)$ and the position of the shock-shock is easily seen, which was the case for all values of $\theta_{w}$ run.

Another interesting case is shock-wave diffraction by a smooth concave wall. In figure 7 we show the calculation for a $30^{\circ}$ circular bend. The shock-shock in this case forms gradually as the shockfront is compressed by the wall. Farther downstream where the wall is straight, the shock-shock moves linearly away from the wall similar to the wedge case. In fact, far downstream the angle between the shock-shock line and the wall is the same as for the $30^{\circ}$ wedge.

Bryson \& Gross (1961) obtained both numerical and experimental results for the diffraction of shock waves by cones, cylinders and spheres. Their numerical solutions also employed the theory of geometrical shock dynamics, but their numerical solutions used the method of characteristics based on (2.7) together with the jump conditions (2.8) and (2.9) in contrast to the more flexible numerical scheme presented here. They found their solutions predicted shockfront and triple-point positions quite well for all their experiments.

As a further test of our numerical scheme, we calculated shockfronts for all the examples presented by Bryson \& Gross. We only display our results for cylinders and spheres as the comparison for cones is essentially the same as for wedges.

We calculate shock-wave diffraction by a cylinder for $M_{0}=2.81$. The results of this calculation are shown in figure 8 . In this case, we use the true $A-M$ relation (3.5) and invert $f(M)$ numerically. This will be done from now on unless specifically stated otherwise. The most interesting feature of this problem is the two loci of shock-shock positions originating from the front and back of the cylinder and continuing downstream. The shock-shock positions are determined numerically by searching for the maximum change in Mach number in the two regions of the shockfront where we know the shock-shock exists. The two loci of shock-shock positions are displayed in figure 8 by the two dashed lines.

In figure 9, we compare the loci of shock-shock positions obtained numerically with experimental data from Bryson \& Gross. We only show representative data points from the clusters of points given by Bryson \& Gross. A sufficient number of points 

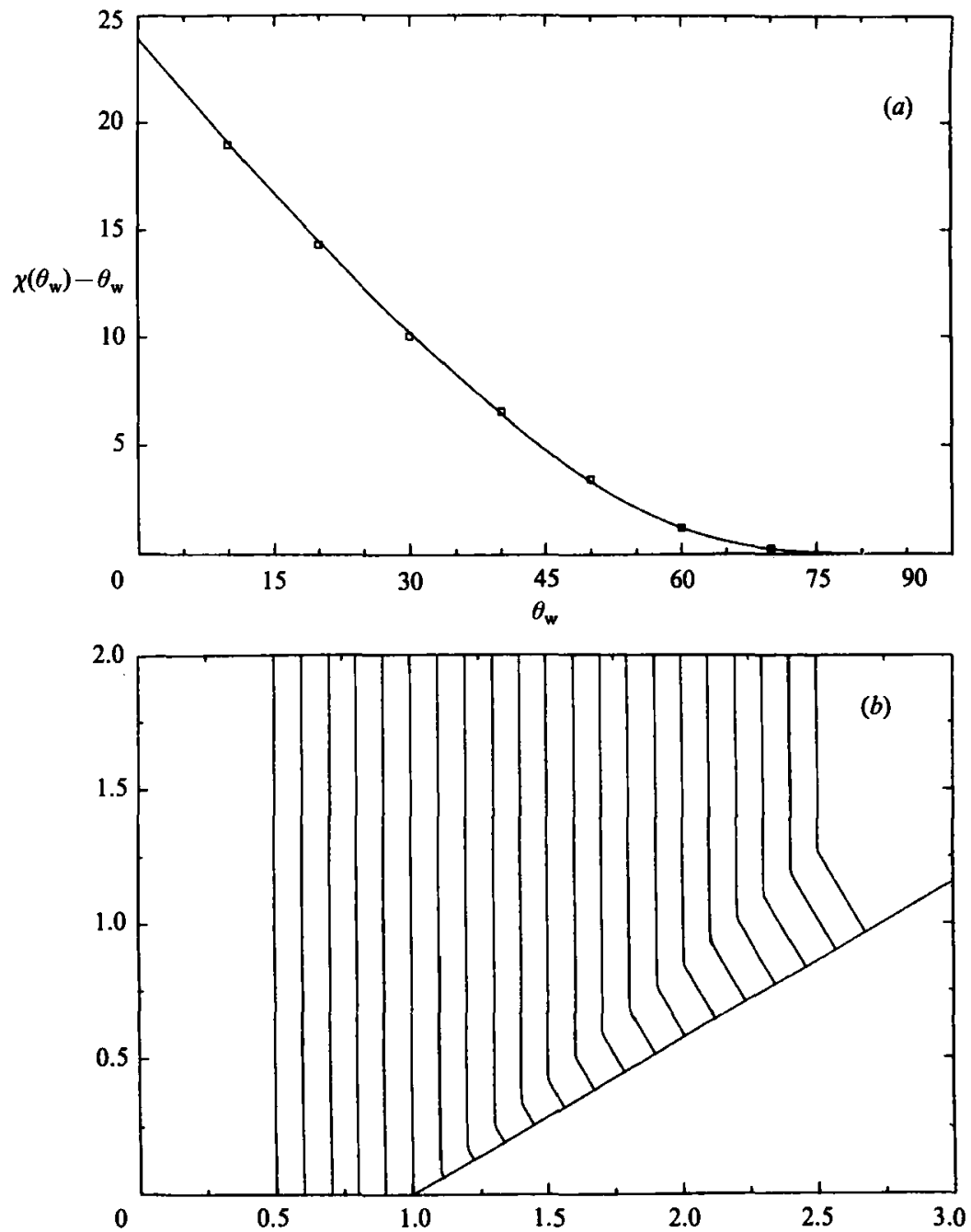

Figure 6. Diffraction by a wedge: $(a) \chi\left(\theta_{\mathrm{w}}\right)-\theta_{\mathrm{w}}$ vs. $\theta_{\mathrm{w}} ;(b)$ shockfronts diffracted by a $30^{\circ}$ wedge.

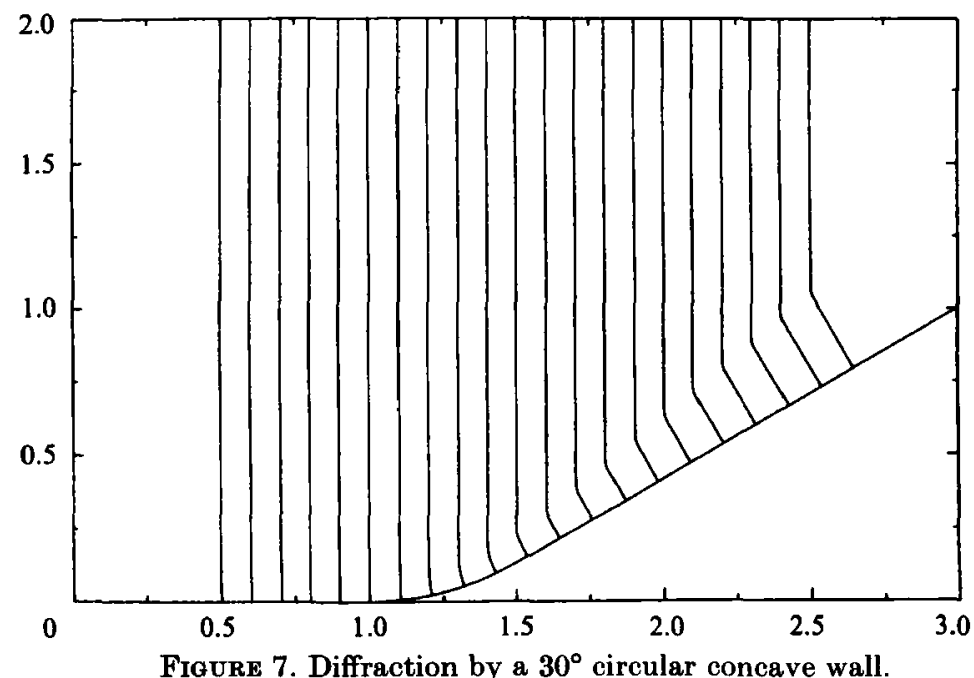




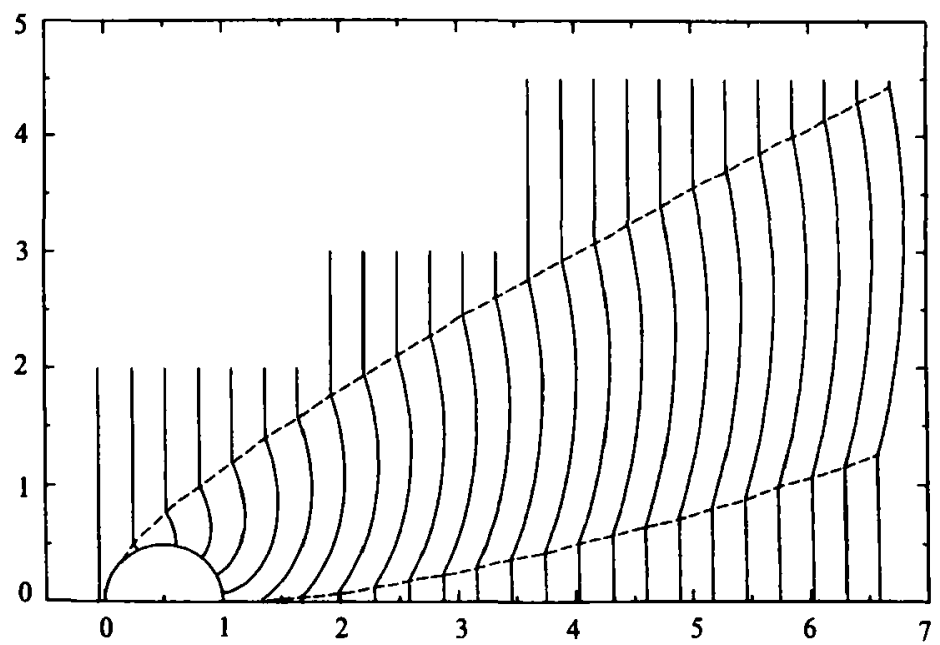

Figure 8. Diffraction by a cylinder for $M_{0}=2.81$. Shockfronts are given by solid lines and loci of shock-shock positions by dashed lines.

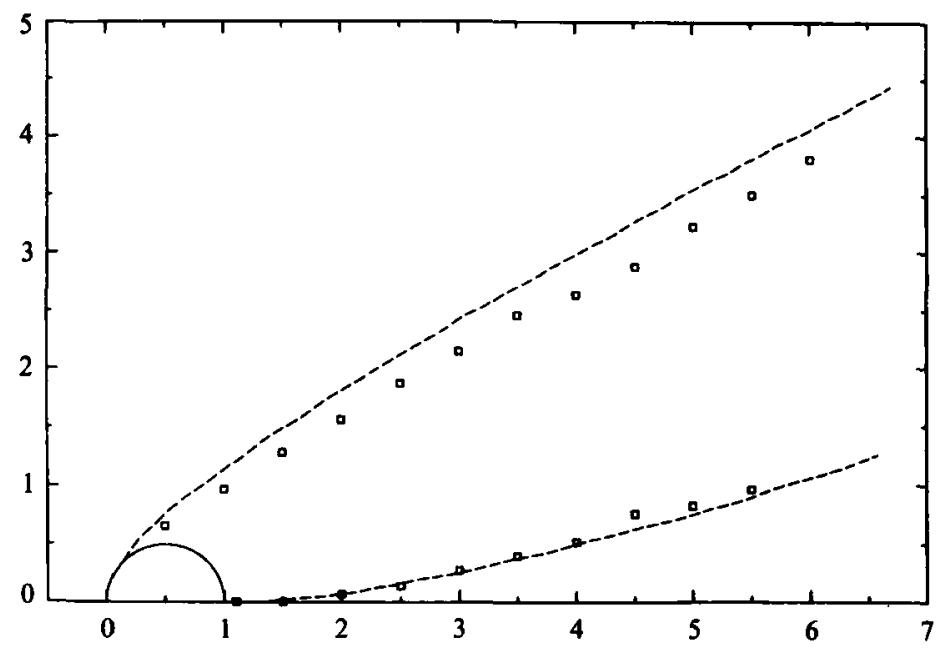

Fiqure 9. Diffraction by a cylinder. Numerical loci of shock-shock positions and experimental triple-point positions.

are displayed in order to indicate a trend in the data. The shock-shock positions calculated numerically are in good agreement with the experimental data for both loci of shock-shock positions. Bryson \& Gross obtained the same agreement using their numerical solution found by the method of characteristics.

Finally, successive shockfronts are calculated for diffraction by a sphere at $M_{0}=2.85$ and are shown in figure 10. For this axisymmetric calculation, the alternative definition of $A_{i}(t)$ given in (3.9) is used in the numerical scheme. The shockfront pattern is much the same as for the cylinder case, and we also calculate the loci of shock-shock positions for the sphere.

The experimental shock-shock positions are shown along with the calculated positions in figure 11. For the sphere, the numerical results predict the experimental data very well for both the front and back loci of shock-shock positions. Bryson \& 


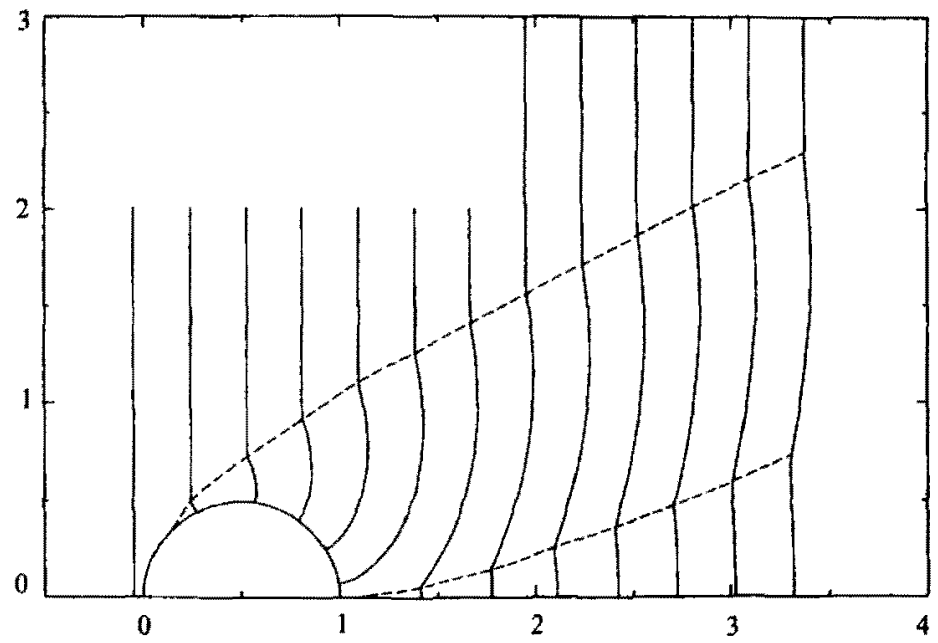

FigURE 10. Diffraction by a sphere for $M_{0}=2.85$. Shockfronts are given by solid lines and loei of shock-shock positions by dashed lines.

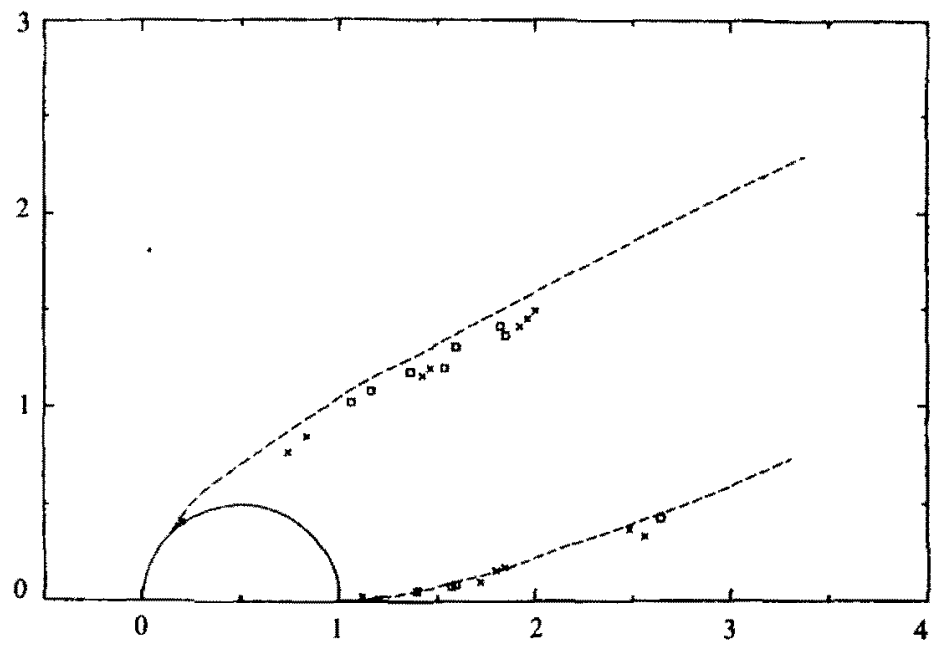

Fiaure 11. Diffraction by a sphere. Numerical loci of shock-shock positions and experimental triple-point positions. $\square, M_{0}=2.85 ; \times, 4.41$.

Gross noted this also for the front locus of shock-shock positions. However, they were unable to obtain a solution using characteristics for the back locus of shock-shock positions. The experimental shock-shock positions for $M_{0}=4.41$ are also shown in figure 11. These data points are also in good agreement with the calculations. This indicates the relative insensitively of the shock-shock positions for high Mach numbers (i.e. strong shocks).

\subsection{Shock waves in channels}

A natural extension of shock-wave diffraction is the case of shock waves in channels. We have seen for shock-wave diffraction that many solutions were available to compare with our numerical solutions. To our knowledge, there are no solutions available for the case of shock waves in channels. The added constraint of an 
additional wall boundary makes solutions obtained using the method of characteristics difficult. This second wall boundary presents no additional problems in our numerical scheme since two boundaries are treated as easily as one.

Edwards et al. (1983) experimentally determined the behaviour of shock waves propagating around $90^{\circ}$ circular bends in channels with nearly rectangular crosssection. Two bends were used, one of inner radius of $48.9 \mathrm{~mm}$ and outer radius $101.1 \mathrm{~mm}$ (channel 1 ), and the other of inner radius $123.9 \mathrm{~mm}$ and outer radius $176.1 \mathrm{~mm}$ (channel 2). The experiments were performed for initial Mach numbers $M_{0}$ between 1.2 and 2.9. The experimental results consisted of a description of the shock-wave pattern in the channels and measurements of wall Mach mumbers $M_{\mathrm{w}}$ on the inner and outer walls.

In figure 12, we display a typical calculation for channel 1 (sharp bend). The shockfront expands around the inner wall in a similar fashion to the convex wall diffraction described in $§ 4.1$. At the outer wall, the shockfront compresses and forms a shock-shock. These two features are essentially independent until the expanding shockfront reaches and shock-shock which is then weakened and turned towards the inner wall. For channel 1, the shock-shock reaches the inner wall in the straight region of the channel after the bend for all Mach numbers considered. The shock-shock reflects off the inner wall and procedes towards the outer wall. This reflection from wall to wall continues for some distance down the channel although it is not displayed in figure 12.

A typical calculation for channel 2 (shallow bend) is shown in figure 13. Many of the features are the same as for channel 1 except that the shock-shock may reach the inner wall before or after the bend, depending on the Mach number. For the particular value of $M_{0}$ shown, the shock-shock is seen to reach the inner wall at the end of the bend. The clear shock-shock reflection observed for channel 1 is not seen here since the angle between the incident shock-shock and the wall is small and the strength of the shock-shock is weaker. For $M_{0}=1.2$, the shock-shock reaches the inner wall after the bend; however, its strength is so weak that a clear reflection is still not observed.

Edwards et al. observed that the shock wave did not recover to a planar front within the bend for channel 1 , which is in agreement with our calculations. Conversely, for channel 2 , they found that recovery to the planar front did occur within the bend. Our calculations tend to support this observation. The shockfront recovers to a nearly planar front in all our calculations for channel 2.

Measurements of $M_{\mathrm{w}}$ versus distance along the wall are given by Edwards et al. for the cases of $M_{0}=1.7,2.1$, and 2.7 for channel 1 and $M_{0}=1.2,1.9$, and 2.9 for channel 2 . These cases are calculated numerically and we determine the values of $\boldsymbol{M}_{\mathbf{w}}$ also. We compare the experimental data with the numerical values in figures 14 and 15. There are two sets of data in each figure corresponding to $M_{\mathrm{w}}$ from each wall. The set with $M_{\mathrm{w}} / M_{0}>1$ comes from the outer wall and the set with $M_{\mathrm{w}} / M_{0}<1$ from the inner wall. For each set, the distance is measured from the beginning of the bend along the corresponding wall with the same scale as used in figures 12 and 13 .

The comparison for channel 1 is shown in figure 14. The Mach number on the inner wall is found experimentally to decrease to $0.6 M_{0}$. We see that the calculations support these measurements. Both numerical and experimental results show the decrease in $M_{\mathrm{w}}$ around the bend and a constant $M_{\mathrm{w}}$ in the straight portion after the bend. On the outer wall, the experimental data show an increase in $M_{w}$ to $1.3 M_{0}$. The shock-dynamics theory predicts a slightly larger increase to $1.4 M_{0}$. Edwards et al. also predict a larger increase using a rough approximation to Whitham's theory 


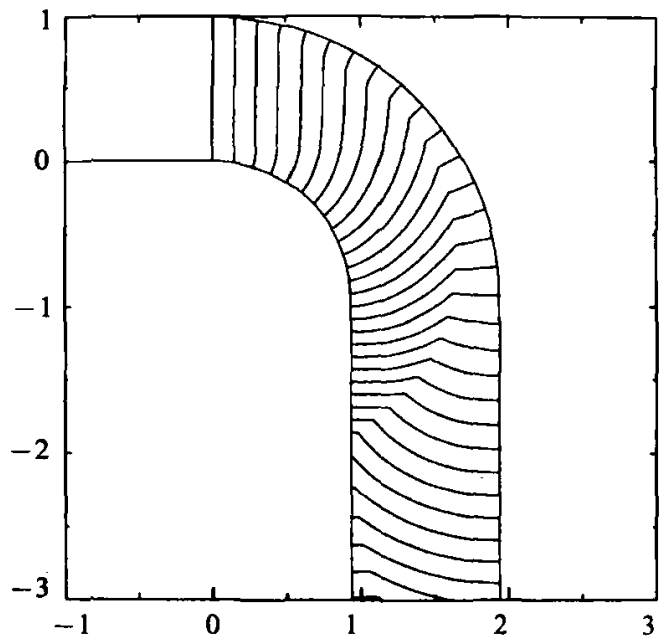

FIGURE 12. Shock waves in channel 1 for $M_{0}=2.1$.

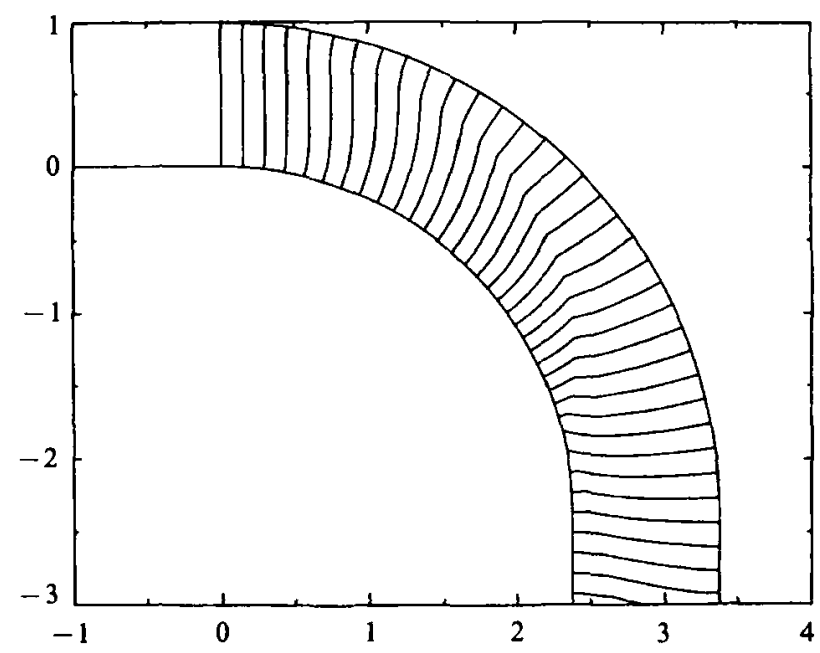

Figure 13. Shock waves in channel 2 for $M_{0}=1.9$.

that ignores the presence of the inner wall, an adequate approximation until the expanding shockfront from the inner wall reaches the shock-shock. In agreement with experiment, we find that the Mach number on the outer wall decreases as a result of this interaction. When the shock-shock reaches the inner wall, a large jump in $M_{w}$ is observed in our calculations. Unfortunately, no experimental measurements were given to compare with this result.

Figure 15 shows the results for channel 2. For the shallow bend, $M_{w}$ decreases to $0.7 M_{0}$ on the inner wall in both experiments and our calculations. $M_{\mathrm{w}}$ increases to $1.15 M_{0}$ on the outer wall in the experiments, whereas our calculations predict a slightly higher value. For $M_{0}=1.2$, the Mach number on the inner wall attains a constant value in the straight portion as in channel 1 . For $M_{0}=1.9$ and 2.9 , a constant value is not reached since the shock-shock meets the inner wall before the end of the bend. The experimental evidence tends to support these observations. 

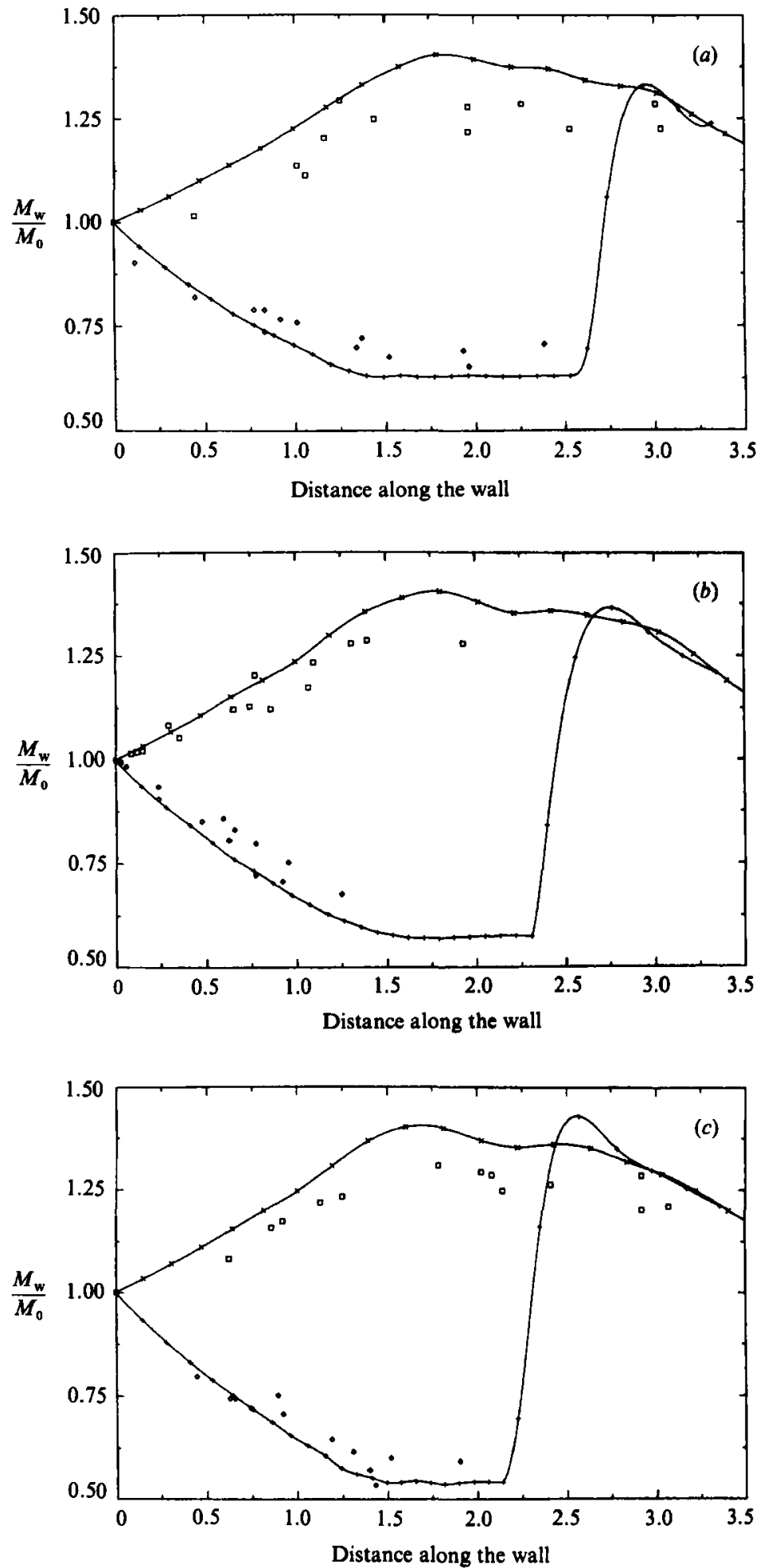

Figure 14. $M_{\mathrm{w}} / M_{0} v s$. distance along the wall for channel $1:$ (a) $M_{0}=1.7 ;(b) M_{0}=2.1 ;$ (c) $M_{0}=2.7 . \square$ and $\diamond$ are experimental points (Edwards et al. 1983), $\times$ and + are our calculated values. 

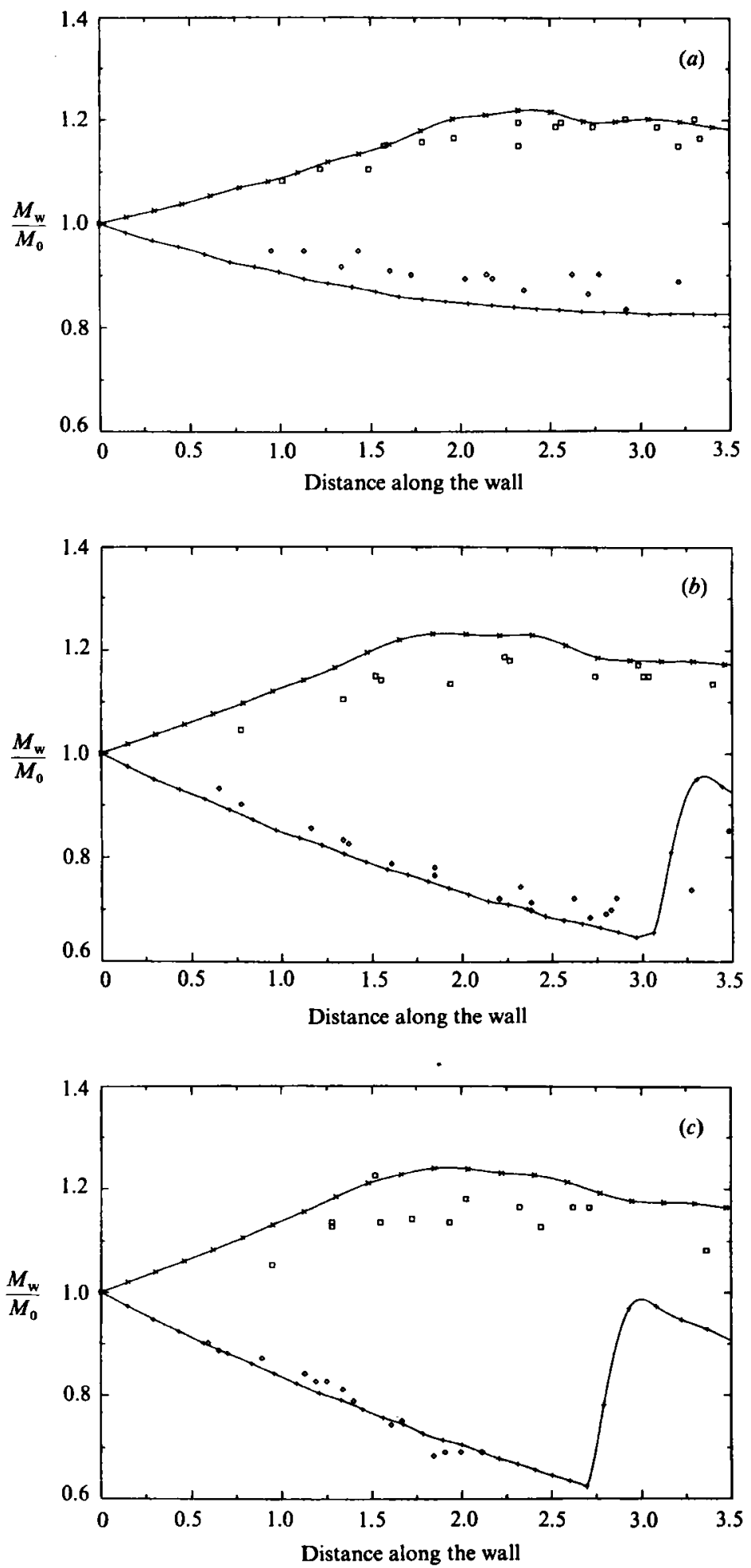

Figure 15. $M_{\mathrm{w}} / M_{0} v s$. distance along the wall for channel $2 ;(a) M_{0}=1.2 ;(b) M_{0}=1.9 ;(c)$ $M_{0}=2.9 . \square$ and $\diamond$ are experimental points (Edwards $e t$ al. 1983), $\times$ and + are our calculated values. 


\subsection{Focusing shock waves}

We are interested in examining the focusing process as determined by geometrical shock dynamics. We numerically experiment with a variety of initial shapes and initial Mach numbers to study how these influences affect the focusing-shock-wave patterns calculated. Most of our calculations are motivated by experiments; however, we also point out other interpretations. Overall, we find reasonable agreement with experimental observations.

Sturtevant \& Kulkarny (1976) experimentally studied the behaviour of focusing weak shock waves. Initially plane shock waves with $M_{0}$ between 1.005 (sound pulse) and 1.5 (comparatively strong shock) were propagated in a shock tube and brought to a focus by reflecting off various concave reflectors at one end of the shock tube. Depending on the initial Mach number of the incident shock wave and minimum radius of curvature of the reflected front, the shock either focused down upon itself and became crossed and folded, as in geometrical acoustics, or the shock did not focus down to a point. In the latter case, a pair of shock-shocks formed with a Mach-stem-like section between the shock-shocks. They found that the crossed pattern was preferred for lower initial Mach numbers or for reflected shockfronts with a smaller minimum radius of curvature.

To compare this experimentally determined behaviour with that predicted by geometrical shock dynamics, two families of initially curved shockfronts were propagated numerically. The first family of initial shockfronts are given by

$$
x=\frac{1}{R}\left(\frac{y^{2}}{y^{2}+2}\right),
$$

where $R$ is the minimum radius of curvature. For $R$ positive, the curve given by (4.9) obtains a minimum of $x=0$ on the axis of symmetry $(y=0)$ and asymptotes to a maximum of $x=1 / R$ for $y \rightarrow \pm \infty$. Successive shockfronts calculated using the one-parameter family in (4.9) as initial conditions give a general picture of the focusing process determined by geometrical shock dynamics. These results also show good qualitative agreement with experiment. We refer to all curves given by (4.9) as profile 1. The second family of initial shockfront shapes labelled profile 2 is displayed in figure 16. The choice of profile 2 was motivated primarily because it compared more closely with experimental data (see later). However, the results for profile 2 may also be viewed in a more general context as an example of a planar front with an inward bulge. The time evolution of these fronts illustrates the stability process for a planar shock wave as given by geometrical shock dynamics.

We first discuss the results obtained using profile 1 . These calculations are performed in the absence of wall boundaries with the free ends of the shockfront initially at $y= \pm 4$. This domain is large enough that the free ends do not affect the focusing process near the axis of symmetry. In the subsequent plots of the shockfronts calculated using profile 1 , we only display the portion of the shockfronts near the axis of symmetry, since the focusing process is of main interest.

In geometrical acoustics, folded wavefronts occur and Sturtevant \& Kulkarny's experiments show that this can continue for weak shockfronts. Geometrical shock dynamics, and the numerical version described here, shows only the leading parts of the fronts. In particular, the numerical point-removal procedure eliminates the overlap. This is done since the removed points would not describe the overlap correctly and would interfere with the continuation of the subsequent numerical steps. To show this, and partially test whether this removal will result in any error 


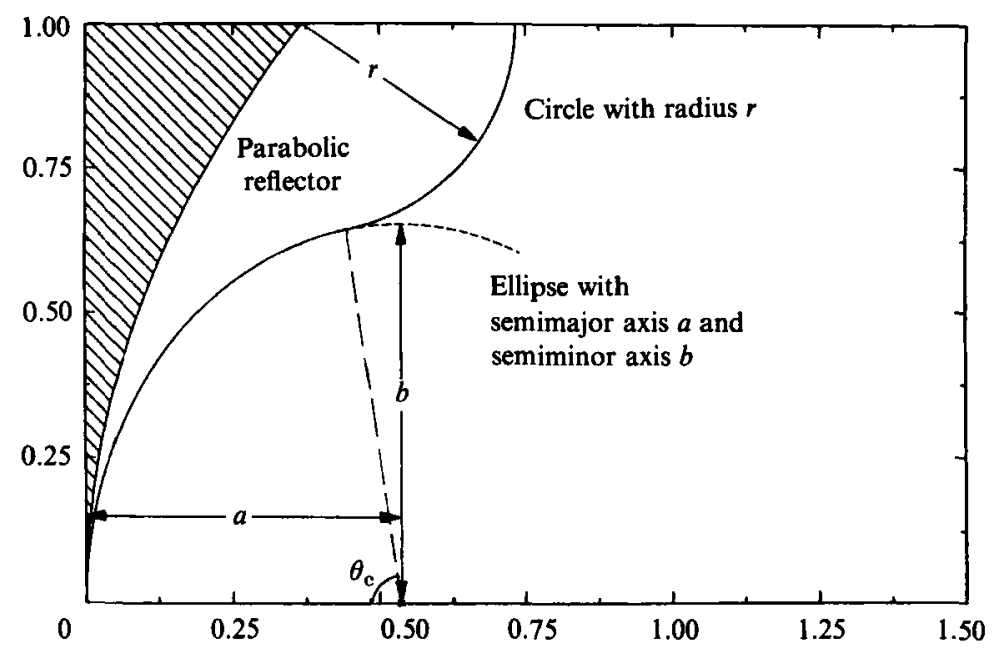

Figure 16. Profile 2 for $b / a=1.22$ and $\theta_{\mathrm{c}}=80^{\circ}$ given by solid curve.

in the calculation of the motion of the shock, the results of the numerical geometrical-shock-dynamics scheme for initial Mach number 1 (a sound wave) were compared with the exact results given by geometrical acoustics.

Figure 17 shows the results for the propagation of a sound wave using geometrical acoustics and shock dynamics. The geometrical-acoustic plot (figure 17a) was obtained directly by drawing the curves (including folds) at various distances out along the normals to the initial curve. The shock-dynamics plot (figure $17 b$ ) was obtained by the numerical scheme using the weak-shock $A-M$ relation with $M_{0}=1$. It can be seen that these curves for the leading fronts are identical. The folds and the caustic do not appear explicitly in the geometrical-shock-dynamics plot. This gives verification that the elimination of the crossed and folded portion of the shockfront does not result in significant error in the remaining portion of the shockfront. The resulting discontinuity in the shockfront corresponds to the regular reflection of the expansion waves propagated along the shock when these waves meet. We note that the theory of geometrical shock dynamics does not explicitly allow for regular reflection and only permits Mach reflection. However, in cases where the Mach stem is very small (in figure $17 b$ for example), we treat these cases as regular reflection.

Successive shockfronts calculated using profile 1 with vatrious values of $R$ and $M_{0}$ are shown in figures 18 and 19 . It can be seen from these plots that geometrical shock dynamics gives the same qualitative behaviour as that observed by Sturtevant \& Kulkarny. Expansion waves form on the shockfront and these move along the front and towards each other. For initial Mach numbers near 1, these expansion waves interact to give regular reflection initially, with Mach reflection occurring and a Mach stem forming at larger times. This change from regular to Mach reflection occurs when the angle between the interacting waves is large enough that regular reflection can no longer occur. For larger initial Mach numbers, no regular reflection occurs and a Mach stem is formed from the start of the interaction. Furthermore, as the minimum radius of curvature of the initial shockfront increases, the transition point to total Mach reflection occurs at lower Mach numbers. It is interesting to note that for Mach numbers as low as 1.3, the behaviour of the shock wave is qualitatively similar to that for strong shocks. 

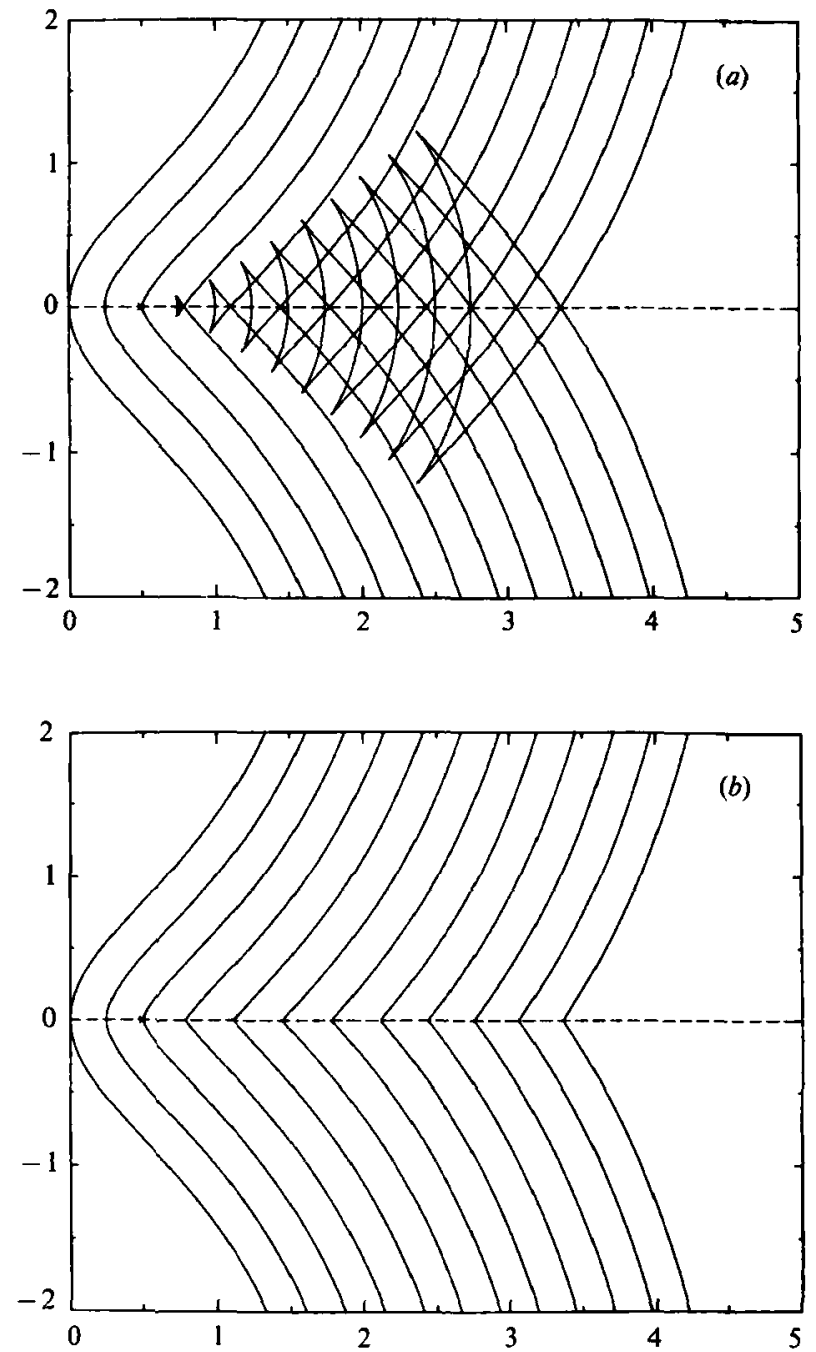

FiguRE 17. Successive shockfronts for profile 1 with $R=0.5$ : (a) geometrical acoustics; (b) geometrical shock dynamics.

A more detailed comparison with the experimental data is obtained using profile 2 (figure 16). In the experiments performed by Sturtevant \& Kulkarny, curved shockfronts were produced by reflecting plane shock waves from a curved reflector. The reflected wave then propagated into the uniform flow entrained by the incident shock. To compare our calculations more closely with these results, we require some initial shockfront shape that approximates the actual curve front as it leaves the reflector surface. The initial shockfront is then propagated numerically into a uniform flow with Mach number $M_{1}$ given by

$$
M_{1}=\frac{2}{\gamma+1} \frac{M_{0}^{2}-1}{M_{0}}
$$

The reflected-shockfront shape as given by geometrical acoustics is a reasonable first approximation to the actual reflected wave for weak incident shocks. For the case of a parabolic reflector, profile 2 with $b / a=1$ is this approximate reflected curve. We 

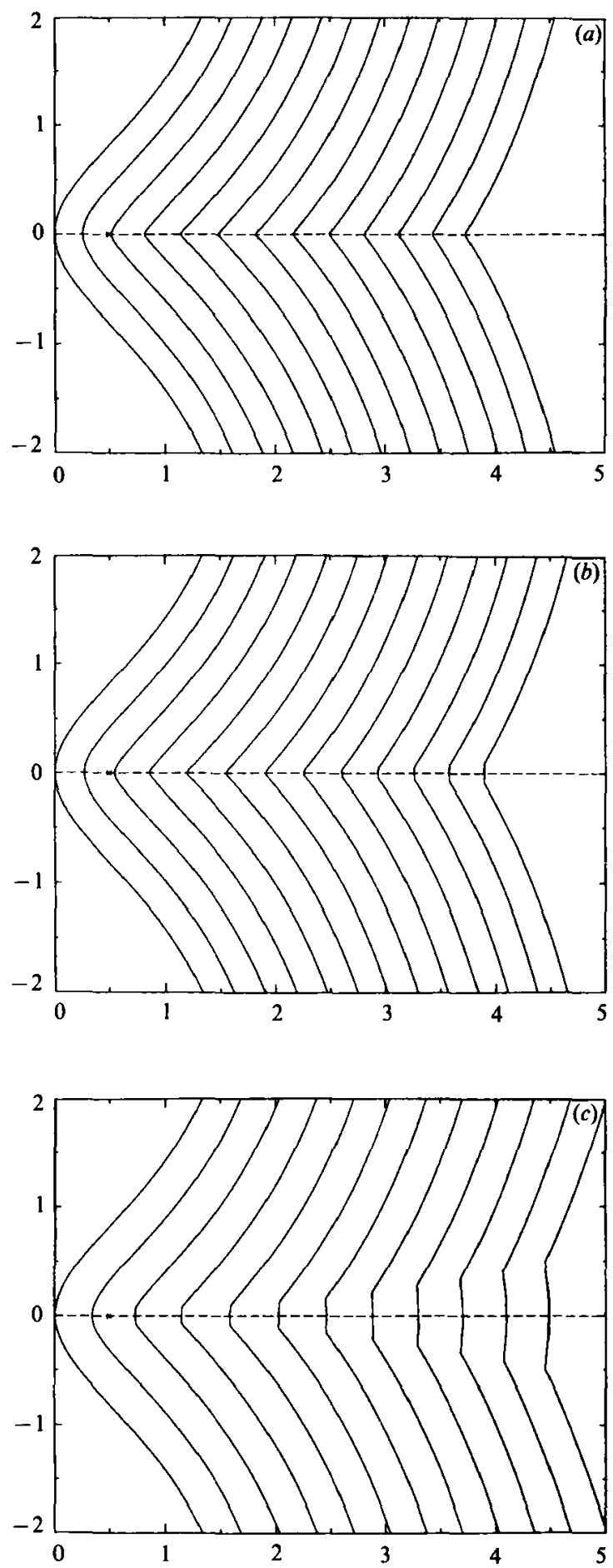

Figure 18. Successive shockfronts for profile 1 and $R=0.5 ;(a) M_{0}=1.02 ;(b) M_{0}=1.06$; (c) $M_{0}=1.30$. 

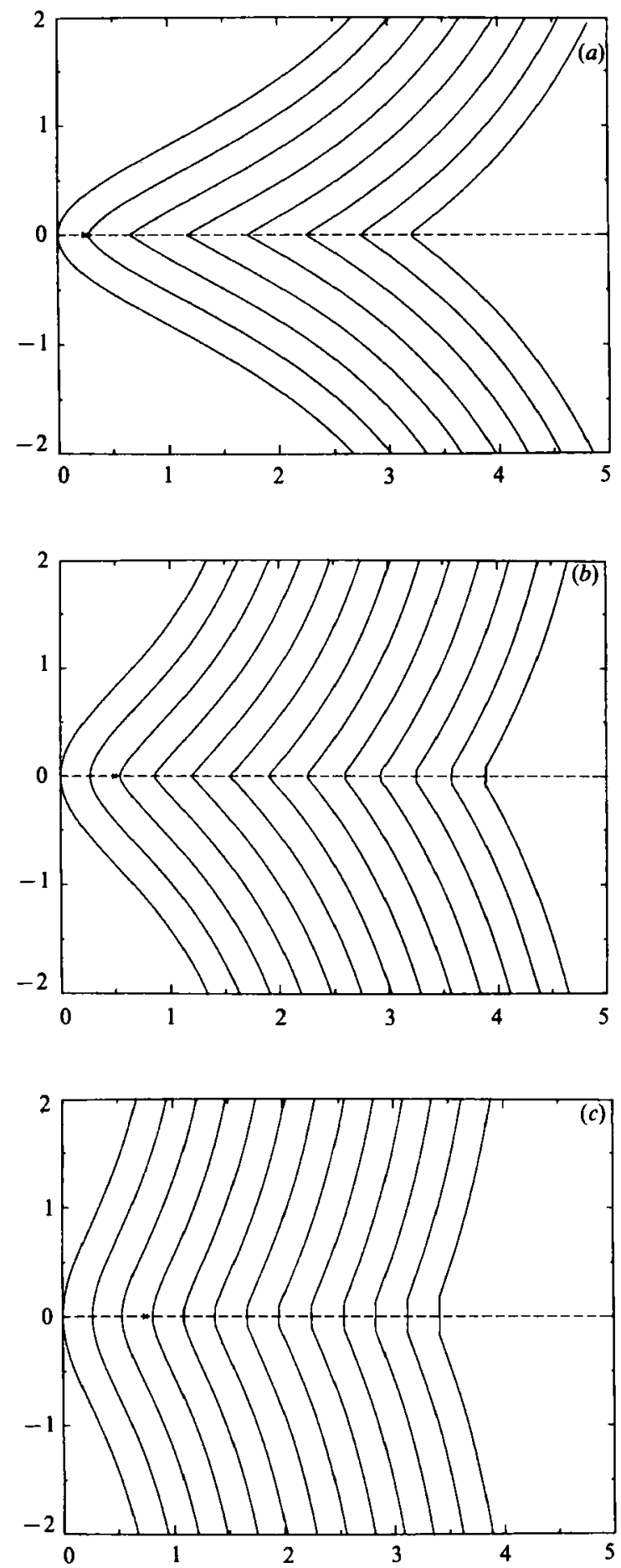

Fraure 19. Successive shockfronts for profile 1 and $M_{0}=1.06$ : (a) $R=0.25 ;(b) R=0.50$; (c) $R=0.75$. 


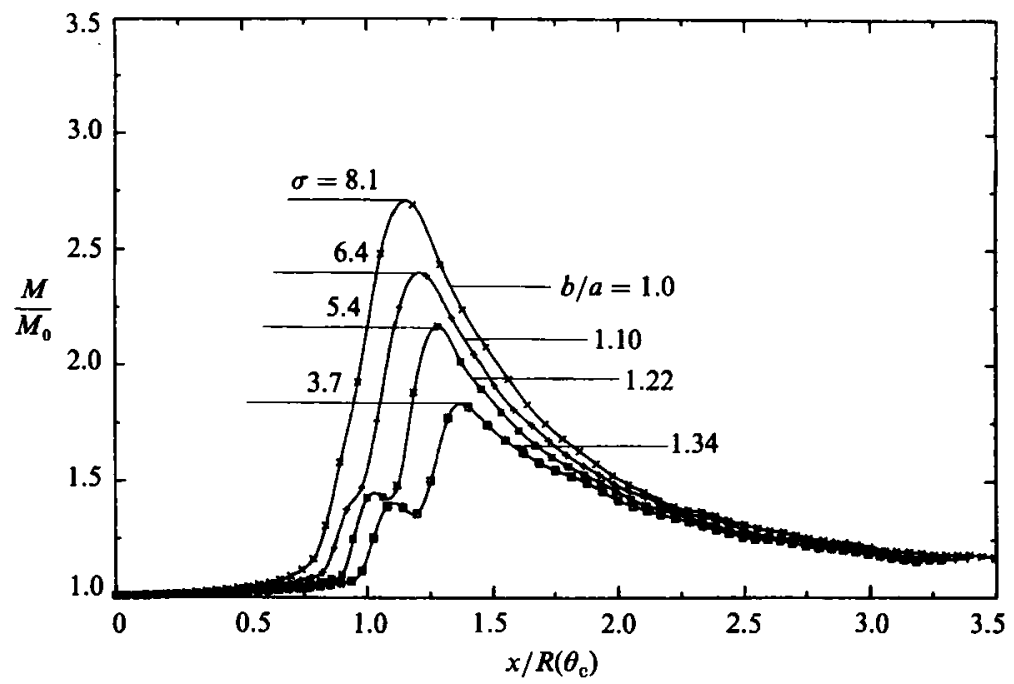

FIqure 20. Mach numbe vs. distance on the axis of symmetry for profile 2 with $\theta_{\mathrm{c}}=80^{\circ}$, $M_{0}=1.1$ and $M_{1}=0.16$.

note, however, that the actual reflected : iock wave has a variable shock strength. The diffracted waves from the corners of the reflector are weaker than the incident shock wave, thus they travel slower than the incident shock. Also, the outer portions of the incident shock wave reflect first and travel back into the flow entrained by the incident shock. These effects combine to give a flatter reflected shock wave than the reflected wave given by geometrical acoustics. The aspect ratio $b / a$ of the ellipse in figure 16 is used to model the flattening of the actual reflected shock wave.

The quantity $b / a$ was found to have a large effect on the peak Mach number near the focus. In figure 20, we plot the Mach number versus distance on the axis of symmetry for different values of $b / a$. For each curve the distance is normalized with respect to the radius of the inner circle $(b / a=1)$ for $\theta_{\mathrm{c}}=80^{\circ}$. As expected the peak Mach number decreases as $b / a$ increases. Sturtevant \& Kulkarny measured a peak relative shock strength $\sigma=4.9$ for $M_{0}=1.1$ and $\theta_{\mathrm{c}}=80^{\circ}$. The relative shock strength is related to the Mach number by

$$
\sigma \equiv \frac{z(M)}{z\left(M_{0}\right)}
$$

where $z(M)$ is the pressure ratio $p_{2} / p_{1}$ of the shock given by

$$
z(M)=1+\frac{2 \gamma}{\gamma+1}\left(M^{2}-1\right)
$$

We see that the initial curve for $b / a=1.25$ gives the best agreement with experiment.

We also studied the effect of a variable initial Mach-number distribution on the peak Mach number. We experimented with different smooth Mach-number distributions. In each case, the initial Mach number on the axis of symmetry was set equal to $M_{0}$ and decayed off the axis to a Mach number greater than or equal to 1 . For weak shocks, our experiments showed a relatively small change in the peak Mach number for a variety of initial distributions.

Some typical views of the focusing-shockfront patterns calculated using profile 2 are shown in figure 21. We found that the general shockfront pictures did not depend 

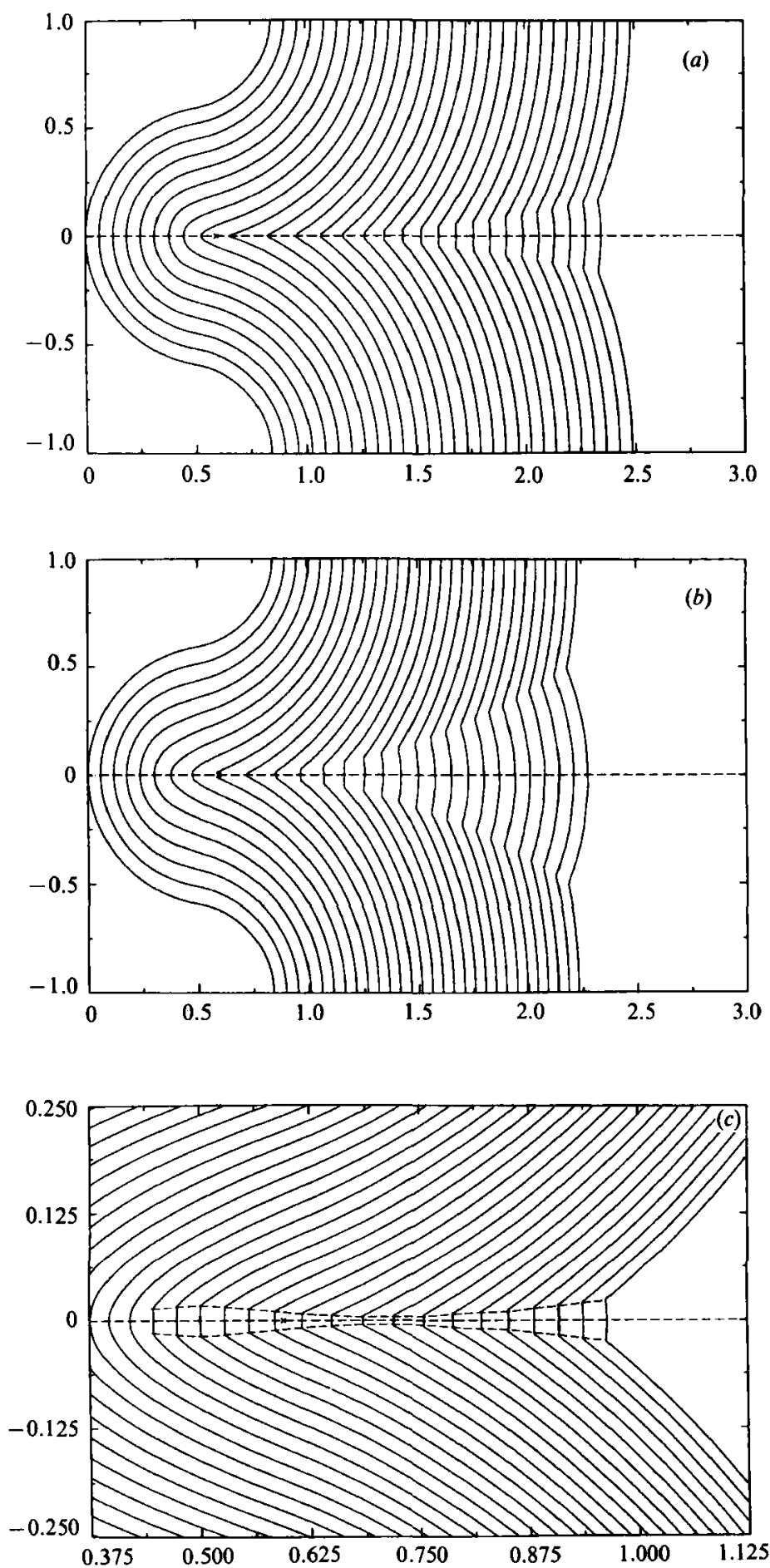

Figure $21(a-c)$. For caption see facing page. 


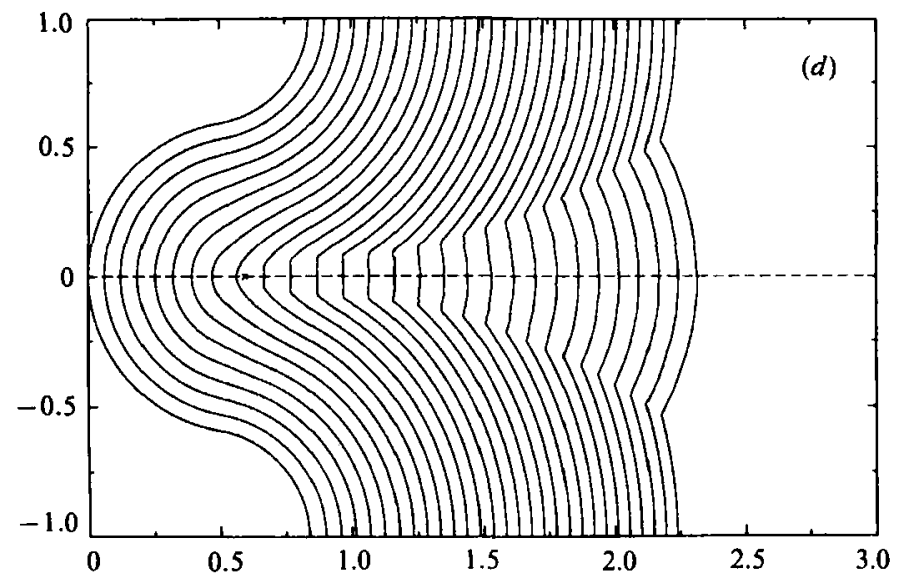

Figure 21. Successive shockfronts for profile 2 with $b / a=1$ and $\theta_{c}=80^{\circ}:(a) M_{0}=1.1$, $M_{1}=0.16 ;$ (b) $M_{0}=1.3, M_{1}=0.44 ;$ (c) $M_{0}=1.3, M_{1}=0.44$ (narrowing Mach stem); $(d)$ $M_{0} \gg 1, M_{1}=0$.

significantly on the quantity $b / a$ or the initial Mach-number distribution. For simplicity, we take $b / a=1$ and $M_{0}=$ constant. Features similar to those seen in figures 18 and 19 are observed in figure 21. For $M_{0}=1.1$, a single shock-shock forms near the focus before a transition to a pair of shock-shocks occurs farther downstream. A slightly larger $M_{0}$ shows a small Mach stem which forms initially just before the focus (figure $21 b$ ). The stem decreases in size as it passes the focus then increases rapidly. This narrowing-Mach-stem phenomenon is clearly seen in figure $21(c)$. Sturtevant \& Kulkarny observed a similar narrowing-Mach-stem phenomenon, although their narrowing occurred for a slightly lower $M_{0}$. We suspect that this difference is a result of the approximate initial conditions.

It is also interesting to note the focusing shockfronts for $M_{0} \gg 1$ (figure $21 d$ ), where we consider the shockfront travelling into a gas at rest $\left(M_{1}=0\right)$. This result is shown for completeness and is not meant to be compared with the experimental data obtained for weak shocks. Here, a Mach stem forms initially and increases in size (without narrowing) as the shockfront propagates downstream.

In all the cases shown in figure 21, the curved shockfront far downstream is approaching a planar front. We see in these examples the stability mechanism for a planar front as determined by geometrical shock dynamics.

A further study of the Mach number versus distance gives added insight into the focusing process for profile 2 . In figure 22 , we see the effect on these curves of varying $M_{0}$ and $\theta_{\mathrm{c}}$. For $b / a=1$, the radius of inner circle is related to $\theta_{\mathrm{c}}$ by

$$
R\left(\theta_{\mathrm{c}}\right)=\frac{1}{2}\left(\frac{\cos \theta_{\mathrm{c}}+1}{\sin \theta_{\mathrm{c}}}\right)
$$

Linear theory (geometrical acoustics) gives a perfect point focus at a distance equal to $R\left(\theta_{\mathrm{c}}\right)$ on the axis of symmetry for profile 2 . The focus position determined by the peak Mach number always occurs after the focus for the nonlinear theory (geometrical shock dynamics). We also observe the formation of a shoulder before the peak in the family of curves for increasing $M_{0}$, in particular for $M_{0}=1.3$. This shoulder corresponds to the initial formation of a Mach stem. For $M_{0}=1.3$, the stem decreases in length as it passes the linear focus point before it eventually increases in length. The peak in Mach number following the shoulder is a result of the narrowing stem. 

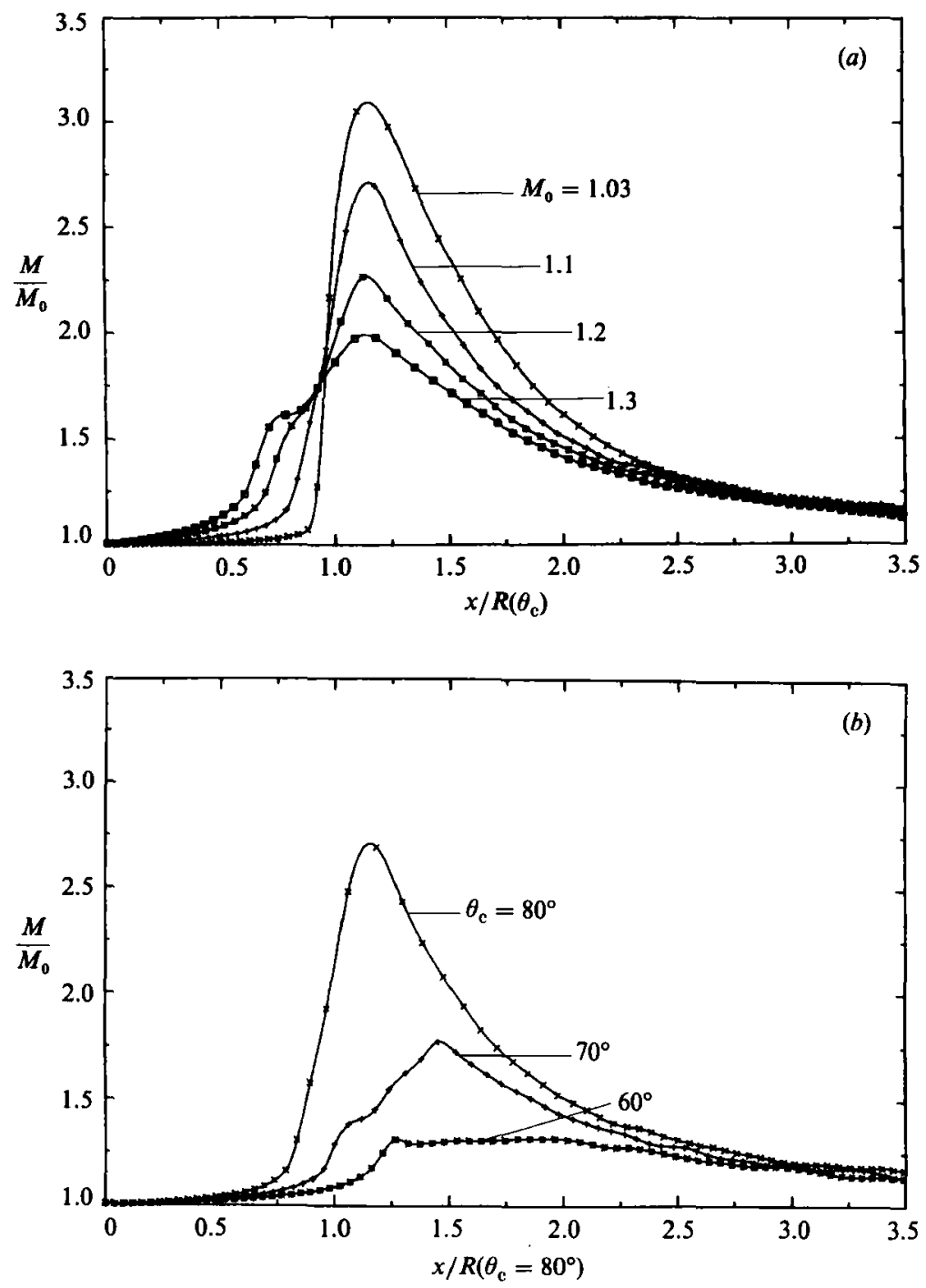

Figure 22. Mach number $v s$. distance on the axis of symmetry for profile 2 with $b / a=1$ :

(a) $\theta_{\mathrm{c}}=80^{\circ} ;$ (b) $M_{0}=1.1, M_{1}=0.16$.

The shoulder formation is a useful feature of the Mach number versus distance curves since it signals the transition from the basic single shock-shock pattern near the focus to the Mach-stem pattern. The three curves in figure $22(b)$ were chosen to show this transition. The curve for $\theta_{\mathrm{c}}=80^{\circ}$ is a typical curve corresponding to the single shock-shock pattern, which we interpret as the actual shock wave being crossed at the focus. The presence of a shoulder following by a peak in Mach number indicates that the focused pattern for $\theta_{\mathrm{c}}=70^{\circ}$ is a transition case. For $\theta_{\mathrm{c}}=60^{\circ}$, the peak has disappeared and only the shoulder remains. In this case, the Mach stem forms initially and propagates downstream without narrowing.

In the present work, we have considered a variety of two-dimensional and axisymmetric problems for the motion of shock waves in gases. The numerical scheme may also be used for other problems where a relation between the amplitude 
and speed of propagation of the wavefront is known, as is the case for soliton motion (Miles 1977) for example. The theory of geometrical shock dynamics also applies for fully three-dimensional wave motion and it should be possible to extend the present numerical scheme to calculate these flows. Other possible considerations are more difficult two-dimensional flows such as shock-wave motion in a variable-density medium. All of these extensions are being considered for future research.

The authors wish to thank Professor G. B. Whitham for many helpful discussions about the work presented in this paper. This research was supported by the ONR (contract numbers N00014-75-C-0702 and N00014-85-K-0403), the DOE (contract number DE-AM03-76SF00767) and the ARO (contract number DAAG29-85-K-0092). The numerical calculations were performed on the Caltech Applied Mathematics IBM 4341 computer and we gratefully acknowledge the IBM corporation for their support.

\section{REFERENCES}

Bryson, A. E. \& Gross, R. W. F. 1961 J. Fluid. Mech. 10, 1-16.

Edwards, D. H., Fearnley, P. \& Nettleton, M. A. 1983 J. Fluid Mech. 132, 257-270.

Miles, J. W. 1977 Z. agnew. Math. Phys., 28, 889-901.

Sturtevant, B. \& Kulkarny, V. A. 1976 J. Fluid Mech. 73, 651-671.

Whitham, G. B. 1957 J. Fluid Mech. 2, 145-171.

Whithaм, G. B. 1959 J. Fluid Mech. 5, 369-386.

Whiтнам, G. B. 1974 Linear and Nonlinear Waves. Wiley. 